\title{
SELECTED ASPECTS OF FUNCTIONING OF THE LAKE GARDNO CATCHMENT (THE WOLIN ISLAND) AS A CHARACTERISTIC INDICATOR OF ENVIRONMENTAL GEOECOSYSTEM CHANGES WITHIN THE COASTAL YOUNG GLACIAL ZONE
}

\author{
Andrzej Kostrzewski, Jacek Tylkowski, Mariusz Samołyk
}

\begin{abstract}
The endorheic catchment of Lake Gardno is located in northwestern Poland on the Wolin Island at a distance of $0.2 \mathrm{~km}$ from the Baltic Sea cliff coast. Within the Lake Gardno catchment, on a monthly basis, water with its quantitative aspects and physicochemical properties was examined at every stage of its circulation. For the diagnosis of the circulation of water and solutes, as well asits mechanisms it was essential to determine the seasonal variability of the concentration of biogenic $\left(\mathrm{NO}_{3}^{-}, \mathrm{NH}_{4}^{+}, \mathrm{K}^{+}\right)$and denudative ions $\left(\mathrm{HCO}_{3}^{-}\right.$, $\left.\mathrm{SO}_{4}^{2-}, \mathrm{Ca}^{2+}, \mathrm{Mg}^{2+}\right)$, and ions supplied primarily from sea aerosols $\left(\mathrm{Cl}^{-}, \mathrm{Na}^{+}\right)$. At the first stage of the water cycle, atmospheric water reaching tree-tops has the lowest mineralization at $1.8 \mathrm{mS} \cdot \mathrm{m}^{-1}$. Throughfall and stemflow lead to an increase in the mineralization up to $8.7 \mathrm{mS} \cdot \mathrm{m}^{-1}$ on average. The next stage, connected with the underground water circulation and leaching of soils, increases the mineralization of water up to $46.3 \mathrm{mS} \cdot \mathrm{m}^{-1}$. The lake water was mineralized at $36.7 \mathrm{mS} \cdot \mathrm{m}^{-1}$. The seasonal variability of the hydrochemical activity of water circulating within the Lake Gardno geoecosystem was represented by groups: with increased concentrations of solutes, with average concentrations of solutes and with reduced concentrations of solutes. The analyzed geoecosystem is characterized by a high concentration level of chloride and sodium ions at every stage of water circulation, which is related to the supply of sea aerosols.
\end{abstract}

Keywords: coastal catchment, Wolin Island, seasonal variability of the water cycle and elements, circulation of water and solutes 


\section{Introduction}

The today's denudation system of the Wolin Island (Kostrzewski 1978, 1993) is conditioned by its location within the temperate climate zone, immediate proximity of the Baltic Sea, early-glacial terrain, land use and anthropopressure. The individual components of the system are based on stable dependencies and cause-effect relationships. Then its dominant dependencies and relationships determine the types of spatial units - geoecosystems in the landscape structure of the Wolin Island. During the last decade the observed climatic changes, increased frequency of extreme processes, anisotropic anthropopressure are additional factors which determine the today's functioning of the denudation system of the Wolin Island.

The concept of geoecosystem and its functioning applied in research proceedings (Kostrzewski 1993), based on methodological and methodical assumptions of empirical science, constitutes a favourable solution for the organization and implementation of research studies on the current state and evolution of the physicogeographical environment. The main objective of the paper is to specify the characteristics of the Lake Gardno catchment connected with the seasonal variability of solutes and their circulation, considerable supplies of biogenic elements in the beech stand and significant loads of solutes coming - together with advection fogs - from over the sea.

The present paper makes an assessment of the environment of the Lake Gardno catchment and its functioning in 2010-2014. The following research objectives were achieved:

- monthly variability of the hydrochemical properties of water circulating;

- seasons of mineralization of water circulating;

- hydrochemical seasons of concentration levels of biogenic ions, denudative ions and ions derived from the supply of sea aerosols;

- load and share levels of biogenic, denudative ions and sea aerosols in the supply of solutes;

- the specificity of the chemical composition of water circulating.

\section{Study area}

Lake Gardno is an important element in the landscape structure of the analyzed geoecosystem (Fig. 1). The Lake Gardno catchment is located within the Wolin Range which is made by the piled-up Wolin End Moraine. The lake area is 2.5 ha and accounts for nearly $1 \%$ of the total catchment area ( $242 \mathrm{ha}$ ). The individuality of forest communities within the Lake Gardno catchment is determined by the 


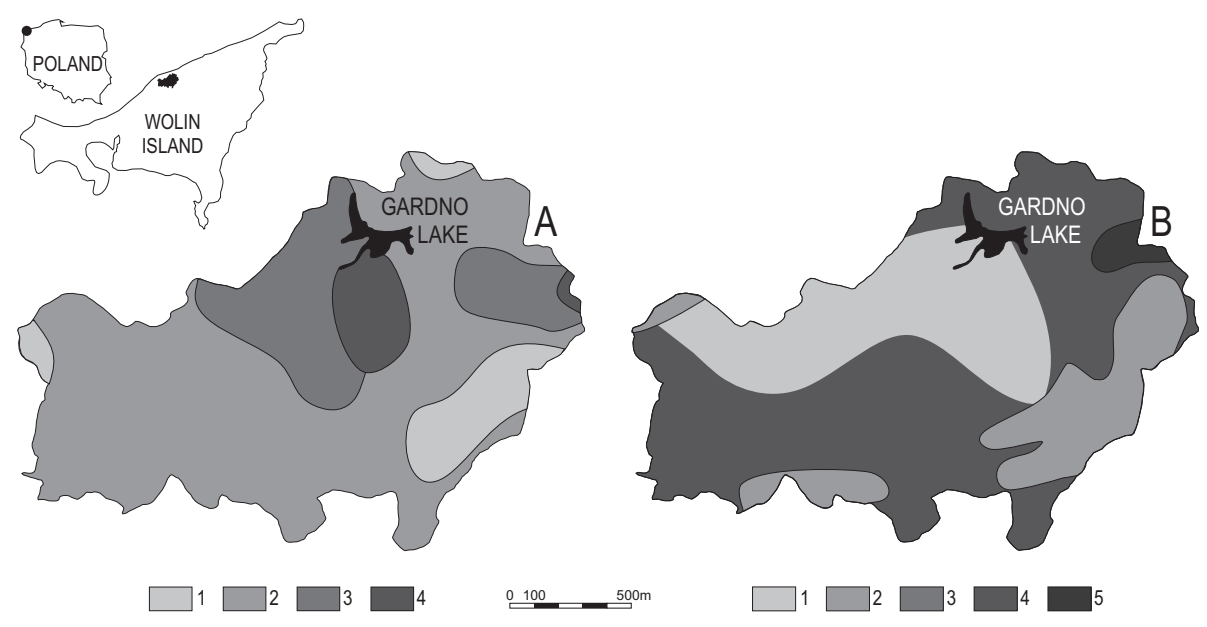

Fig. 1. Location of the research area with lithology and soils types

A. Lithology of surface quaternary formations (Gościńska 2003, changed): 1 - very fine sand, 2 - fine sand, 3 - medium sand, 4 - glacial till.

B. Soils types (Adamczewska, Jewasińska 1986; Borowiec 1969, changed): 1 - Endoeutric Cambisols and poorly developed Podzols, 2 - Dystric Cambisols, 3 - Dystric Cambisols and Endoeutric Cambisols, 4 - moderately developed Podzols, 5 - well developed Podzols.

Source: authors' own study.

presence of Pomeranian beeches (Piotrowska 1955), the catchment is almost completely forested (Kostrzewski et al. 2015; Tylkowski, Samołyk 2015).

The lake is fed by atmospheric and ground water. Choiński (1978) states that this is probably the only lake in Poland which is drained into the sea. On the Wolin Island - in connection with the geological structure - underground watersheds presumably may not match surface watersheds.

The examined catchment is characterized by well-permeable surface deposits in lithological terms and they determine the water cycle. The good permeability of surface deposits results in a very poor hydrographical network. Apart from Lake Gardno there are no hydrographical objects within the examined geoecosystem. It is dominated by deep infiltration of precipitation and supplies of underground aquifers. Surface deposits within the Lake Gardno catchment are represented mainly by fine-grained sands (Fig. 1A). The share of medium-grained fractions increases with depth. According to Gościńska (2003) the variability of subsurface sediments is low. Along a cross-section of the catchment area, at a deep of $0.5 \mathrm{~m}$, 
sandy sediments such as fine-grained $(82 \%)$ and medium-grained $(6 \%)$ sands are most common. Below this depth their lithological variability increases. Sandy deposits still dominate, although clay deposits increase their share. Horizontally, the largest area is covered by fine-grained sands. The area located south of Lake Gardno and the eastern part of the catchment is made of clay.

The relief and lithogenic structure determine soil formation. The research area of Lake Gardno catchment is dominated by Albic Alisols and Albic Brunic Arenosols (Komisarek et al. 2010). Soils (their type and structure) are linked with forest habitats (their types), parent material and weather conditions regulated - to a large extent - by the coastal location of the catchment (Fig. 1B). Soils are acidic, which significantly determines the composition of the sorption complex and the level of saturation with basic cations. There is a well-formed forest floor with a thickness up to about $10 \mathrm{~cm}$ in the soils of the catchment. This forest floor is little permeable to water which hinders precipitation being infiltrated into the ground. Soils in the immediate vicinity of the lake are characterized by very acidic reaction; $\mathrm{pH}$ values (in $\mathrm{KCl}$ ) for the forest floor range from 2.57 to 3.39 , for $\mathrm{AE}$ as well as Es and Bhs horizons - from 2.52 to 3.34 (Komisarek et al. 2010).

The lithological, pedological and topoclimatic features of the Lake Gardno catchment determine the individuality of the examined geoecosystem in the landscape structure of the Wolin Island. This individuality is - first and foremost defined by: its coastal location within the temperate climatic zone, the domination of forests, the surfacially endorheic nature of the catchment and the associated circulation of water as well as the relatively low anthropopressure.

\section{Materials and methods}

The Lake Gardno catchment has been the subject of research in the form of reviews as well as detailed geomorphological, topoclimatic (Bogucki, Tamulewicz 1992; Tylkowski, Samołyk 2010; Tylkowski 2012, 2013), hydrological (Choiński 1978), pedological (Borowiec 1969; Prusinkiewicz 1971) and tourism-related studies.

The regularities presented in the paper are based on the analysis of information and data gathered under the Integrated Environmental Monitoring Programme (Kostrzewski 1993; Kostrzewski et al. 2006, 2015; Kolander, Tylkowski 2008) implemented at some test areas within the Lake Gardno catchment.

The natural individuality of the catchment (Kostrzewski et al. 2011, 2015) and the implemented Integrated Environmental Monitoring program contributed to the development of numerous papers, mainly analytical in their character, on the circulation of water and matter within the Lake Gardno catchment. The research topics of the developed papers were mainly focused to present the variability of 
chemical components in water circulating in the catchment on an annual basis (Kolander 2005; Kolander, Tylkowski 2007; Kolander et al. 2008; Tylkowski et al. 2008) and seasonal changes in the supply of biogenic elements into the catchment (Tylkowski et al. 2009). The basic quantitative characteristics representing different stages within the water cycle in the Lake Gardno catchment were obtained.

Water at the atmospheric stage was collected for hydrochemical analyses based on the Hellman pluviometer (Kolander, Tylkowski 2007, 2008; Kostrzewski et al. 2011, 2015; Kruszyk et al. 2015). Atmospheric precipitation was collected at a midforest clearing in the Lake Gardno catchment. Throughfall was measured with 10 pluviometers arranged in triangles, 10 meters away from one another. Stemflow was measured at 3 trees. Additionally, an experimental collector of fog-generated sediments was placed within the discussed area. It was possible to measure the level and quality of throughflow by means of a gatherer mounted on a hill (its slope) adjacent to Lake Gardno. In reference to the lithological structure, earthworks aimed to collect water migrating along the slope were performed. Within the lower part of the slope at a depth of $60 \mathrm{~cm}$ a gatherer collecting soil-through water was embedded. The water was directed to a specially-adapted pluviometer. Water from the lithospheric stage was also subjected to constant monitoring. All changes in the level of underground water were recorded by a water level recorder. Samples of underground water were taken by a probe at a depth of about 2 meters. The lake water was collected at a depth of $0.5 \mathrm{~m}$ below the lake surface. All the measuring instruments were equipped with loggers recording water levels on a ten-minute basis.

\section{Results}

The research results based on long-term data provided in the present paper are aimed to verify the research studies conducted so far in the Lake Gardno catchment.

Within the Lake Gardno geoecosystem at the first stage of the water cycle, atmospheric water reaching tree-tops has the lowest mineralization at $15 \mathrm{mg} \cdot \mathrm{dm}^{-3}$ (Kostrzewski et al. 2011, 2015). Throughfall and stemflow lead to an increase in the mineralization up to $80 \mathrm{mg} \cdot \mathrm{dm}^{-3}$ on average. The next stage connected with the underground water circulation and leaching of soils increases the mineralization of water up to $195 \mathrm{mg} \cdot \mathrm{dm}^{-3}$. In contrast, the lake water is mineralized at $185 \mathrm{mg} \cdot \mathrm{dm}^{-3}$. The $\mathrm{pH}$ of water circulating in the catchment (2009) is characterized - and that should be stressed - by a decrease in acidification at the further stages of water circulation in the catchment ( $\mathrm{pH} 4.78$ in the open field, $\mathrm{pH} 6.47$ - lake water). A seasonal variability in the mineralization of water within the Lake Gardno catchment (namely increased in the cool half-year and reduced in the warm 
half-year) was found. Fogs with their frequent occurrence emphasize the geographical individuality of Lake Gardno catchment. In 2009 fogs were present for 39 days and provided $91 \mathrm{~mm}$ of water (15\% of the total vertical precipitation). The chemical composition of fog-generated precipitation is a distinguishing characteristic, thus it can be singled out as a separate hydrochemical type of water. The chemical composition and high mineralization of fog-generated sediments is the closest to sea water. The paper on the singling-out of hydrochemical seasons in the Lake Gardno catchment, which highlights its individuality, is synthetic in its character (Kolander, Tylkowski 2008).

\section{Monthly variability of the hydrochemical properties of water circulation}

For the diagnosis of the circulation of water and solutes and its mechanisms it is essential to define the seasonal variability of concentration of biogenic $\left(\mathrm{NO}_{3}^{-}\right.$, $\left.\mathrm{NH}_{4}^{+}, \mathrm{K}^{+}\right)$and denudative ions $\left(\mathrm{HCO}_{3}^{-}, \mathrm{SO}_{4}^{2-}, \mathrm{Ca}^{2+}, \mathrm{Mg}^{2+}\right)$ and ions supplied primarily from sea aerosols $\left(\mathrm{Cl}^{-}, \mathrm{Na}^{+}\right)$.

The temporal variability in the level of these ions in the mineralization of water circulating in the Lake Gardno catchment showed higher annual variability levels of biogenic ions compared to ions derived from the supply of sea aerosols and especially in reference to the most stable denudative ions. In spatial terms, ahigher annual variability in the level of various solutes in the total mineralization of water referred to the atmospheric (rather than lithospheric) stage of the water cycle. Therefore, it can be assumed that the highest annual variability characterized the concentration level of biogenic ions, especially nitrates and potassium in precipitation in the open field and stemflow. Then, the highest annual stability characterized the concentration level of denudative ions, especially magnesium and sulphates in underground and lake water (Fig. 2).

As for the spatial variability, an increase in the mineralization and $\mathrm{pH}$ of water was found at subsequent stages of water circulation. The atmospheric system of water circulation (atmospheric precipitation, throughfall, stemflow) was characterized by a high concentration level of biogenic ions and ions supplied by sea aerosols. Within water reaching the forest bottom the concentration level of denudative ions was relatively low. The concentration level of denudative ions significantly increased in the course of water migration through the slope covers down to underground water and further to the lake reservoir. At the underground stage of water circulation the concentration level of biogenic ions was much lower than the one in the course of infiltration of precipitation through the beech stand. The analysed geoecosystem was characterized by a high concentration level of chloride 

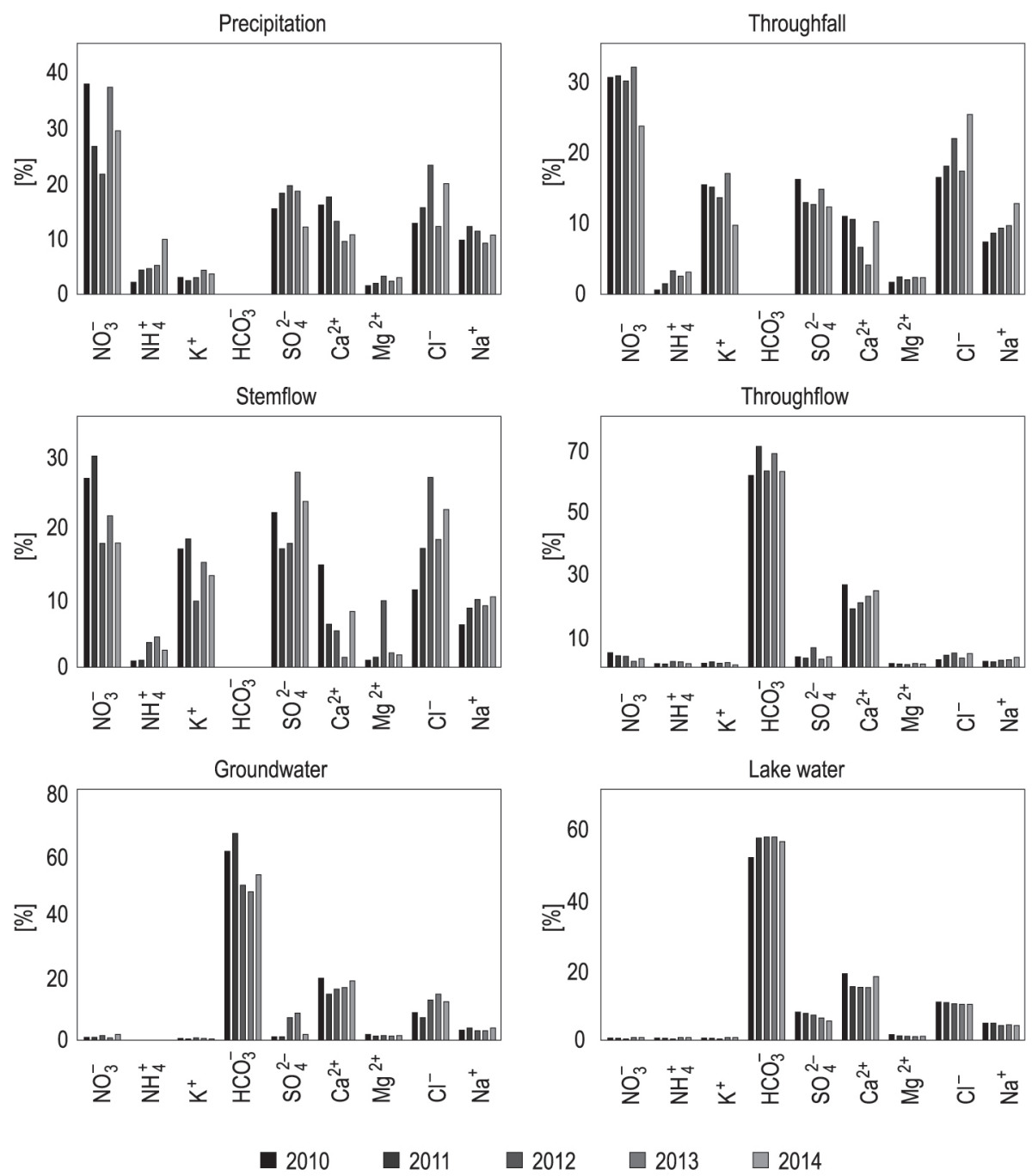

Fig. 2. Annual variability of biogenic $\left(\mathrm{NO}_{3}^{-}, \mathrm{NH}_{4}^{+}, \mathrm{K}^{+}\right)$, denudative $\left(\mathrm{HCO}_{3}^{-}, \mathrm{SO}_{4}^{2-}, \mathrm{Ca}^{2+}\right.$, $\left.\mathrm{Mg}^{2+}\right)$ ions and ions from the supply of sea aerosols $\left(\mathrm{Cl}^{-}, \mathrm{Na}^{+}\right)$in water circulating in the Lake Gardno catchment (2010-2014) in a beech stand

Source: authors' own study. 
and sodium ions at every stage of the water circulation, which is related to the supply of sea aerosols. Within the examined coastal early-glacial geoecosystem at every stage of the water circle among biogenic ions nitrates had the highest concentration. As for denudative ions at the atmospheric stage of the water cycle, sulphate ions had the highest concentration and at the lithospheric stage - bicarbonate ions. Then, among ions derived mainly from the supply of sea aerosols, chlorides had the highest concentration (Tab. 1, 2).

Table. 1. Monthly variability of the hydrochemical properties of water circulating at the atmospheric stage of the water cycle in the Lake Gardno catchment in a beech stand (2010-2014)

\begin{tabular}{|c|c|c|c|c|c|c|c|c|c|c|c|}
\hline \multirow{3}{*}{ Month } & \multirow{2}{*}{$\begin{array}{l}\text { Water } \\
\text { quantity }\end{array}$} & \multirow{2}{*}{$\begin{array}{c}\text { Conductivity } \\
\text { SEC }\end{array}$} & \multirow{2}{*}{$\frac{\text { Reaction }}{\mathrm{pH}}$} & \multicolumn{3}{|c|}{ Biogenic ions } & \multicolumn{3}{|c|}{ Denudation ions } & \multicolumn{2}{|c|}{ Sea aerosols } \\
\hline & & & & $\mathrm{NO}_{3}^{-}$ & $\mathrm{NH}_{4}^{+}$ & $\mathrm{K}^{+}$ & $\mathrm{SO}_{4}^{2-}$ & $\mathrm{Ca}^{2+}$ & $\mathrm{Mg}^{2+}$ & $\mathrm{Cl}^{-}$ & $\mathrm{Na}^{+}$ \\
\hline & {$[\mathrm{mm}]$} & {$\left[\mathrm{mS} \cdot \mathrm{m}^{-1}\right]$} & {$[-]$} & \multicolumn{8}{|c|}{$\left[\mathrm{mg} \cdot \mathrm{dm}^{-3}\right]$} \\
\hline \multicolumn{12}{|c|}{ Precipitation } \\
\hline$X I$ & 56.3 & 1.73 & 5.48 & 3.00 & 0.37 & 0.28 & 1.36 & 1.43 & 0.17 & 1.06 & 1.04 \\
\hline XII & 51.4 & 1.69 & 5.21 & 1.05 & 0.32 & 0.24 & 1.42 & 1.55 & 0.58 & 5.03 & 2.27 \\
\hline I & 49.8 & 1.52 & 5.37 & 2.40 & 0.20 & 0.12 & 1.06 & 0.93 & 0.14 & 1.13 & 0.83 \\
\hline II & 30.5 & 1.80 & 5.84 & 3.42 & 0.35 & 0.13 & 2.00 & 1.44 & 0.14 & 1.13 & 0.71 \\
\hline III & 23.1 & 2.91 & 5.78 & 7.07 & 0.91 & 0.30 & 2.03 & 1.48 & 0.23 & 1.61 & 1.13 \\
\hline IV & 29.6 & 2.72 & 6.20 & 5.90 & 0.84 & 0.22 & 1.75 & 2.07 & 0.16 & 1.14 & 0.84 \\
\hline V & 52.6 & 2.39 & 6.37 & 6.95 & 0.79 & 0.48 & 1.83 & 1.43 & 0.25 & 1.62 & 1.16 \\
\hline VI & 69.2 & 1.42 & 5.88 & 3.77 & 0.25 & 0.35 & 1.74 & 1.42 & 0.23 & 1.38 & 0.83 \\
\hline VII & 102.2 & 1.77 & 4.96 & 3.71 & 0.37 & 0.24 & 1.48 & 1.13 & 0.19 & 1.32 & 0.77 \\
\hline VII & 94.8 & 1.18 & 5.35 & 1.86 & 1.12 & 0.47 & 1.44 & 0.98 & 0.23 & 1.23 & 0.85 \\
\hline IX & 48.1 & 1.56 & 5.33 & 1.48 & 1.33 & 0.60 & 1.52 & 1.13 & 0.26 & 1.17 & 0.85 \\
\hline$x$ & 54.1 & 1.54 & 5.22 & 1.75 & 0.50 & 0.25 & 1.10 & 1.17 & 0.21 & 1.78 & 1.04 \\
\hline$X I-X$ & 661.8 & 1.85 & 5.58 & 3.53 & 0.61 & 0.31 & 1.56 & 1.34 & 0.23 & 1.63 & 1.03 \\
\hline \multicolumn{12}{|c|}{ Throughfall } \\
\hline$X I$ & 33.8 & 6.72 & 6.36 & 4.61 & 0.25 & 8.33 & 5.16 & 3.59 & 0.82 & 9.42 & 3.21 \\
\hline XII & 30.8 & 6.49 & 5.88 & 6.50 & 0.32 & 7.36 & 7.90 & 3.67 & 1.63 & 20.65 & 9.60 \\
\hline I & 29.9 & 5.15 & 6.24 & 5.92 & 0.40 & 2.08 & 3.79 & 1.67 & 0.74 & 7.60 & 3.35 \\
\hline II & 18.3 & 6.89 & 5.78 & 8.92 & 0.79 & 1.98 & 5.45 & 2.61 & 0.80 & 6.77 & 3.12 \\
\hline III & 13.9 & 7.55 & 5.80 & 8.07 & 1.33 & 1.42 & 4.48 & 2.88 & 0.57 & 8.99 & 3.63 \\
\hline IV & 17.8 & 11.44 & 6.19 & 14.87 & 1.09 & 2.75 & 7.60 & 3.40 & 1.03 & 11.48 & 5.90 \\
\hline V & 31.6 & 8.52 & 6.86 & 16.62 & 0.52 & 7.03 & 4.89 & 3.77 & 1.35 & 5.90 & 2.96 \\
\hline
\end{tabular}


SElected aspects of functioning of the Lake Gardno catchment...

\begin{tabular}{|c|c|c|c|c|c|c|c|c|c|c|c|}
\hline \multirow{3}{*}{ Month } & \multirow{2}{*}{$\begin{array}{l}\text { Water } \\
\text { quantity }\end{array}$} & \multirow{2}{*}{$\begin{array}{c}\text { Conductivity } \\
\text { SEC }\end{array}$} & \multirow{2}{*}{$\frac{\text { Reaction }}{\mathrm{pH}}$} & \multicolumn{3}{|c|}{ Biogenic ions } & \multicolumn{3}{|c|}{ Denudation ions } & \multicolumn{2}{|c|}{ Sea aerosols } \\
\hline & & & & $\mathrm{NO}_{3}^{-}$ & $\mathrm{NH}_{4}^{+}$ & $\mathrm{K}^{+}$ & $\mathrm{SO}_{4}^{2-}$ & $\mathrm{Ca}^{2+}$ & $\mathrm{Mg}^{2+}$ & $\mathrm{Cl}^{-}$ & $\mathrm{Na}^{+}$ \\
\hline & {$[\mathrm{mm}]$} & {$\left[\mathrm{mS} \cdot \mathrm{m}^{-1}\right]$} & {$[-]$} & \multicolumn{8}{|c|}{$\left[\mathrm{mg} \cdot \mathrm{dm}^{-3}\right]$} \\
\hline VI & 41.5 & 6.24 & 6.50 & 12.55 & 0.76 & 4.39 & 3.90 & 2.28 & 0.83 & 4.00 & 2.21 \\
\hline VII & 61.3 & 5.09 & 6.56 & 17.75 & 1.33 & 3.25 & 3.89 & 3.94 & 0.68 & 3.17 & 1.89 \\
\hline VII & 56.9 & 6.69 & 6.41 & 13.33 & 0.56 & 3.02 & 3.71 & 2.29 & 0.74 & 4.18 & 2.03 \\
\hline IX & 28.9 & 7.46 & 6.98 & 9.45 & 1.20 & 5.80 & 4.64 & 3.63 & 0.81 & 5.01 & 2.39 \\
\hline $\mathrm{x}$ & 32.5 & 9.39 & 6.99 & 5.35 & 1.07 & 11.89 & 4.37 & 2.53 & 0.89 & 9.58 & 3.02 \\
\hline$X I-X$ & 397.1 & 7.30 & 6.38 & 10.33 & 0.80 & 4.94 & 4.98 & 3.02 & 0.91 & 8.06 & 3.61 \\
\hline \multicolumn{12}{|c|}{ Stemflow } \\
\hline$X I$ & 3.9 & 5.78 & 5.79 & 10.00 & 0.63 & 7.97 & 10.51 & 4.62 & 0.85 & 9.21 & 3.59 \\
\hline XII & 3.7 & 13.00 & 5.45 & 7.77 & 0.79 & 8.23 & 19.42 & 6.22 & 4.04 & 16.22 & 6.62 \\
\hline I & 3.5 & 14.04 & 5.68 & 9.00 & 0.76 & 8.87 & 27.21 & 6.71 & 3.54 & 25.66 & 9.29 \\
\hline II & 2.4 & 11.56 & 5.83 & 3.46 & 0.81 & 5.23 & 12.44 & 2.81 & 1.90 & 15.68 & 5.85 \\
\hline III & 2.0 & 17.00 & 5.76 & 19.73 & 3.38 & 8.10 & 27.66 & 2.80 & 3.22 & 27.95 & 11.27 \\
\hline IV & 2.4 & 17.04 & 5.71 & 12.90 & 2.47 & 8.60 & 20.70 & 1.76 & 2.66 & 21.30 & 11.63 \\
\hline V & 3.8 & 7.81 & 5.67 & 11.91 & 1.26 & 7.52 & 5.18 & 3.51 & 1.69 & 8.14 & 4.32 \\
\hline VI & 4.7 & 4.81 & 6.21 & 5.98 & 1.31 & 4.63 & 3.46 & 2.43 & 0.87 & 5.10 & 2.30 \\
\hline VII & 6.5 & 6.05 & 6.21 & 11.42 & 1.12 & 5.74 & 4.31 & 2.57 & 0.89 & 3.50 & 1.93 \\
\hline VII & 6.2 & 9.48 & 6.25 & 17.49 & 1.38 & 7.27 & 7.09 & 4.36 & 1.61 & 5.87 & 2.32 \\
\hline IX & 3.5 & 6.51 & 5.41 & 11.60 & 1.77 & 6.09 & 5.87 & 2.63 & 1.44 & 3.70 & 1.78 \\
\hline$x$ & 3.7 & 7.86 & 6.26 & 12.22 & 1.81 & 9.04 & 9.38 & 3.21 & 1.33 & 5.67 & 2.38 \\
\hline$X I-X$ & 46.3 & 10.08 & 5.85 & 11.12 & 1.46 & 7.28 & 12.77 & 3.64 & 2.00 & 12.33 & 5.27 \\
\hline
\end{tabular}

Source: authors' own study.

Table 2. Monthly variability of the hydrochemical properties of water circulating at the lithospheric stage of the water cycle in the Lake Gardno catchment in a beech stand (2010-2014)

\begin{tabular}{|c|c|c|c|c|c|c|c|c|c|c|c|c|}
\hline \multirow{3}{*}{ Month } & \multirow{2}{*}{$\begin{array}{c}\text { Conductivity } \\
\text { SEC }\end{array}$} & \multirow{2}{*}{$\begin{array}{c}\begin{array}{c}\text { Reac- } \\
\text { tion }\end{array} \\
\mathrm{pH}\end{array}$} & \multicolumn{4}{|c|}{ Biogenic ions } & \multicolumn{4}{|c|}{ Denudation ions } & \multicolumn{2}{|c|}{ Sea aerosols } \\
\hline & & & $\mathrm{PO}_{4}^{3-}$ & $\mathrm{NO}_{3}^{-}$ & $\mathrm{NH}_{4}^{+}$ & $\mathrm{K}^{+}$ & $\mathrm{SO}_{4}^{2-}$ & $\mathrm{Ca}^{2+}$ & $\mathrm{Mg}^{2+}$ & $\mathrm{HCO}_{3}^{-}$ & $\mathrm{Cl}^{-}$ & $\mathrm{Na}^{+}$ \\
\hline & {$\left[\mathrm{mS} \cdot \mathrm{m}^{-1}\right]$} & {$[-]$} & {$\left[\mu \mathrm{g} \cdot \mathrm{dm}^{-3}\right]$} & \multicolumn{9}{|c|}{$\left[\mathrm{mg} \cdot \mathrm{dm}^{-3}\right]$} \\
\hline \multicolumn{13}{|c|}{ Throughflow [60 cm depth] } \\
\hline$X I$ & 22.70 & 7.63 & 0.07 & 2.51 & 1.06 & 0.15 & 8.21 & 50.90 & 0.64 & 118.98 & 12.54 & 3.69 \\
\hline XII & 23.16 & 7.09 & 0.31 & 6.60 & 0.88 & 0.57 & 10.04 & 48.76 & 0.95 & 126.92 & 15.49 & 4.01 \\
\hline I & 18.08 & 7.74 & 0.11 & 2.65 & 1.86 & 0.30 & 3.79 & 39.98 & 0.70 & 106.48 & 7.94 & 3.47 \\
\hline II & 17.42 & 7.79 & 0.02 & 0.71 & 1.03 & 0.30 & 4.44 & 39.38 & 0.96 & 111.97 & 4.69 & 3.66 \\
\hline
\end{tabular}




\begin{tabular}{|c|c|c|c|c|c|c|c|c|c|c|c|c|}
\hline \multirow{3}{*}{ Month } & \multirow{2}{*}{$\begin{array}{c}\text { Conductivity } \\
\text { SEC }\end{array}$} & \multirow{2}{*}{$\begin{array}{c}\begin{array}{c}\text { Reac- } \\
\text { tion }\end{array} \\
\mathrm{pH}\end{array}$} & \multicolumn{4}{|c|}{ Biogenic ions } & \multicolumn{4}{|c|}{ Denudation ions } & \multicolumn{2}{|c|}{ Sea aerosols } \\
\hline & & & $\mathrm{PO}_{4}{ }^{3-}$ & $\mathrm{NO}_{3}^{-}$ & $\mathrm{NH}_{4}^{+}$ & $\mathrm{K}^{+}$ & $\mathrm{SO}_{4}^{2-}$ & $\mathrm{Ca}^{2+}$ & $\mathrm{Mg}^{2+}$ & $\mathrm{HCO}_{3}^{-}$ & $\mathrm{Cl}^{-}$ & $\mathrm{Na}^{+}$ \\
\hline & {$\left[\mathrm{mS} \cdot \mathrm{m}^{-1}\right]$} & {$[-]$} & {$\left[\mu \mathrm{g} \cdot \mathrm{dm}^{-3}\right]$} & \multicolumn{9}{|c|}{$\left[\mathrm{mg} \cdot \mathrm{dm}^{-3}\right]$} \\
\hline III & 19.96 & 7.66 & 0.25 & 5.92 & 0.40 & 0.82 & 4.99 & 38.58 & 0.88 & 125.09 & 6.90 & 3.74 \\
\hline IV & - & - & - & - & - & - & - & - & - & - & - & - \\
\hline V & 22.14 & 7.27 & 0.41 & 11.04 & 1.52 & 0.84 & 5.31 & 46.56 & 0.96 & 149.90 & 5.75 & 3.60 \\
\hline $\mathrm{VI}$ & 20.09 & 7.52 & 0.17 & 7.51 & 1.68 & 0.79 & 6.41 & 45.16 & 0.81 & 120.82 & 5.56 & 3.38 \\
\hline VII & 19.11 & 7.37 & 0.03 & 3.26 & 1.61 & 0.42 & 4.31 & 41.02 & 0.78 & 142.58 & 3.80 & 2.59 \\
\hline VII & 14.39 & 6.75 & 1.16 & 5.76 & 0.98 & 0.52 & 4.34 & 40.59 & 0.79 & 94.58 & 2.92 & 3.02 \\
\hline IX & - & - & - & - & - & - & - & - & - & - & - & - \\
\hline$X$ & 19.40 & 7.72 & 0.06 & 2.90 & 1.85 & 0.38 & 4.94 & 41.79 & 0.71 & 118.68 & 8.48 & 3.48 \\
\hline $\mathrm{XI}-\mathrm{X}$ & 19.64 & 7.45 & 0.26 & 4.89 & 1.28 & 0.51 & 5.68 & 43.27 & 0.83 & 121.60 & 7.41 & 3.46 \\
\hline \multicolumn{13}{|c|}{ Groundwater [200 cm depth] } \\
\hline $\mathrm{XI}$ & 45.03 & 7.26 & 0.58 & 5.33 & 0.20 & 1.95 & 9.17 & 75.17 & 8.57 & 236.89 & 51.53 & 17.71 \\
\hline XII & 53.93 & 7.08 & 0.51 & 4.28 & 0.14 & 1.88 & 28.69 & 76.75 & 9.06 & 223.45 & 64.13 & 17.71 \\
\hline I & 51.01 & 7.27 & 1.11 & 13.92 & 0.23 & 1.75 & 32.62 & 77.78 & 8.01 & 228.13 & 55.19 & 15.65 \\
\hline II & 45.65 & 6.97 & 0.41 & 7.09 & 0.15 & 1.80 & 19.22 & 73.72 & 8.71 & 228.00 & 52.58 & 19.29 \\
\hline III & 45.20 & 7.50 & 0.24 & 2.78 & 0.10 & 1.49 & 14.54 & 58.19 & 7.54 & 210.11 & 45.51 & 15.77 \\
\hline IV & 41.78 & 7.45 & 0.28 & 3.06 & 0.54 & 1.70 & 11.70 & 65.05 & 8.11 & 224.81 & 46.17 & 16.88 \\
\hline V & 42.41 & 7.40 & 0.73 & 4.62 & 0.15 & 2.28 & 8.74 & 62.90 & 7.10 & 241.87 & 43.62 & 28.60 \\
\hline VI & 43.94 & 7.52 & 0.57 & 4.21 & 0.23 & 1.71 & 7.65 & 69.94 & 8.27 & 243.82 & 48.87 & 17.50 \\
\hline VII & 44.24 & 7.02 & 0.25 & 4.10 & 0.26 & 1.66 & 14.77 & 72.91 & 8.34 & 234.18 & 50.69 & 17.14 \\
\hline VII & 44.40 & 7.05 & 0.30 & 4.19 & 0.17 & 1.64 & 13.18 & 73.27 & 8.27 & 232.64 & 52.27 & 17.13 \\
\hline IX & 44.03 & 7.25 & 0.31 & 4.13 & 0.20 & 1.72 & 27.86 & 81.40 & 10.84 & 237.48 & 55.80 & 18.11 \\
\hline$x$ & 53.60 & 6.94 & 0.35 & 12.90 & 0.45 & 2.37 & 29.78 & 83.69 & 9.18 & 226.25 & 56.24 & 17.98 \\
\hline$X I-X$ & 46.27 & 7.23 & 0.47 & 5.88 & 0.24 & 1.83 & 18.16 & 72.56 & 8.50 & 230.64 & 51.88 & 18.29 \\
\hline \multicolumn{13}{|c|}{ Lake water } \\
\hline XI & 36.70 & 7.56 & 0.11 & 1.47 & 0.16 & 1.55 & 23.96 & 56.76 & 6.41 & 187.53 & 35.86 & 16.05 \\
\hline XII & 37.19 & 7.63 & 0.18 & 1.35 & 0.15 & 1.41 & 21.70 & 53.87 & 5.86 & 187.96 & 33.12 & 16.74 \\
\hline I & 33.67 & 7.53 & 0.12 & 1.19 & 0.21 & 1.66 & 24.02 & 60.26 & 6.68 & 177.64 & 38.92 & 17.56 \\
\hline II & 40.04 & 7.39 & 0.15 & 1.02 & 0.15 & 1.54 & 20.26 & 55.45 & 5.69 & 187.08 & 34.06 & 15.20 \\
\hline III & 39.53 & 7.75 & 0.13 & 1.66 & 0.15 & 1.44 & 19.66 & 49.07 & 5.21 & 164.82 & 30.88 & 13.72 \\
\hline IV & 34.58 & 7.86 & 0.09 & 0.67 & 0.17 & 1.45 & 23.94 & 57.76 & 6.18 & 171.74 & 37.33 & 16.44 \\
\hline V & 37.33 & 7.87 & 0.12 & 0.76 & 0.13 & 1.32 & 24.28 & 55.75 & 6.18 & 173.90 & 37.93 & 16.68 \\
\hline VI & 36.03 & 8.05 & 0.16 & 0.59 & 0.14 & 1.19 & 23.70 & 53.67 & 6.16 & 169.50 & 37.55 & 16.83 \\
\hline
\end{tabular}


Selected aspects of functioning of the Lake Gardno catchment...

\begin{tabular}{|c|c|c|c|c|c|c|c|c|c|c|c|c|}
\hline \multirow{3}{*}{ Month } & Conductivity & Reac- & \multicolumn{4}{|c|}{ Biogenic ions } & \multicolumn{4}{|c|}{ Denudation ions } & \multicolumn{2}{|c|}{ Sea aerosols } \\
\hline & SEC & $\mathrm{pH}$ & $\mathrm{PO}_{4}{ }^{3-}$ & $\mathrm{NO}_{3}^{-}$ & $\mathrm{NH}_{4}^{+}$ & $\mathrm{K}^{+}$ & $\mathrm{SO}_{4}^{2-}$ & $\mathrm{Ca}^{2+}$ & $\mathrm{Mg}^{2+}$ & $\mathrm{HCO}_{3}^{-}$ & $\mathrm{Cl}^{-}$ & $\mathrm{Na}^{+}$ \\
\hline & {$\left[\mathrm{mS} \cdot \mathrm{m}^{-1}\right]$} & {$[-]$} & {$\left[\mu \mathrm{g} \cdot \mathrm{dm}^{-3}\right]$} & \multicolumn{9}{|c|}{$\left[\mathrm{mg} \cdot \mathrm{dm}^{-3}\right]$} \\
\hline VII & 34.75 & 7.95 & 0.22 & 0.91 & 0.15 & 1.20 & 23.54 & 51.42 & 6.12 & 171.82 & 36.73 & 16.60 \\
\hline VII & 35.90 & 7.86 & 0.23 & 0.45 & 0.21 & 1.27 & 24.16 & 54.75 & 6.28 & 173.29 & 36.56 & 16.48 \\
\hline IX & 37.13 & 7.76 & 0.12 & 0.67 & 0.17 & 1.27 & 23.24 & 48.86 & 6.37 & 176.76 & 37.15 & 16.47 \\
\hline$x$ & 37.46 & 7.61 & 0.08 & 0.42 & 0.17 & 1.45 & 23.16 & 57.11 & 6.26 & 190.13 & 36.63 & 16.50 \\
\hline $\mathrm{XI}-\mathrm{X}$ & 36.69 & 7.74 & 0.14 & 0.93 & 0.16 & 1.40 & 22.97 & 54.56 & 6.12 & 177.68 & 36.06 & 16.27 \\
\hline
\end{tabular}

Source: authors' own study.

\section{Hydrochemical seasons of water circulating}

At every stage of the water cycle the seasonal variability of water mineralization and concentrations of solutes was determined. Seasons of hydrochemical activity were singled out by means of statistical methods under the cluster analysis. With the Euclidean distance and single-bond method applied in the cluster analysis, a hierarchical clustering of months was made in terms of water mineralization and concentration of biogenic, denudative ions and ions coming mainly from the supply of sea aerosols (Fig. 3). The seasonal variability of hydrochemical activity of water circulating within the Lake Gardno geoecosystem was represented by 3 groups: A - with increased concentrations of solutes, $\mathrm{B}$ - with average concentrations of solutes and $\mathrm{C}$ - with reduced concentrations of solutes (Tab. 3).

Water migrating in the Lake Gardno catchment is characterized by a high variability of seasons of its mineralization. In the case of atmospheric precipitation reaching the tree-top zone the highest mineralization took place in spring, in March and May (14.63 $\mathrm{mg} \cdot \mathrm{dm}^{-3}$ on average). Then, the season of reduced mineralization of atmospheric precipitation lasted much longer -8 months - from June to November and in January and February. The average concentration of solutes in atmospheric precipitation was $8.55 \mathrm{mg} \cdot \mathrm{dm}^{-3}$ at that time. In the case of throughfall precipitation the season of high water mineralization occurred in December only with the average content of solute ions at $57.64 \mathrm{mg} \cdot \mathrm{dm}^{-3}$. In the case of throughfall, its season of reduced water mineralization, with an average concentration level of solutes at $33.41 \mathrm{mg} \cdot \mathrm{dm}^{-3}$, lasted for 10 months, except December and April. In the case of stemflow its season of increased hydrochemical activity occurred in March $\left(104.11 \mathrm{mg} \cdot \mathrm{dm}^{-3}\right)$ and its season of reduced concentration of solute ions lasted 8 months, mainly in summer and autumn $\left(40.50 \mathrm{mg} \cdot \mathrm{dm}^{-3}\right)$. In underground water its season of high mineralization occurred in autumn-winter months, from September to October and from December to January, when the 

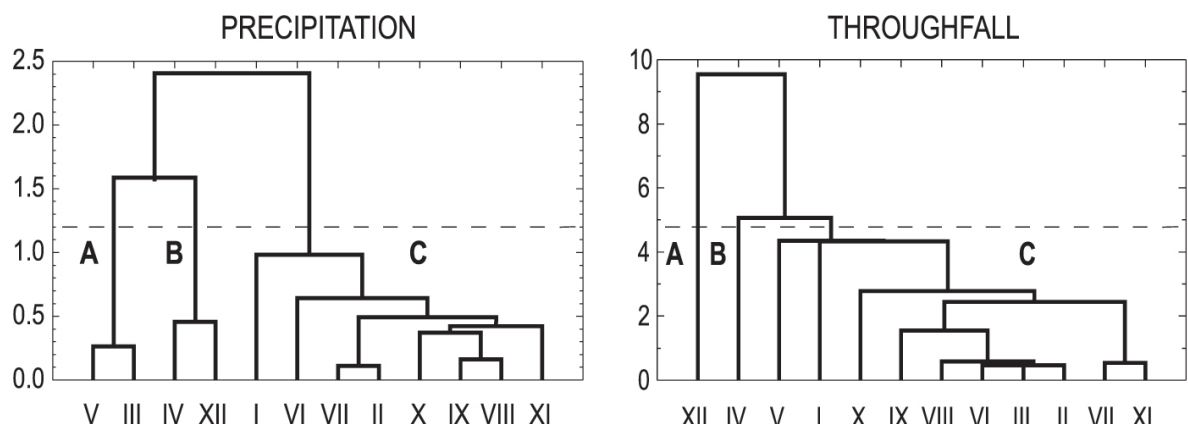

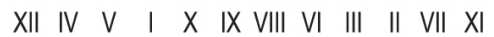
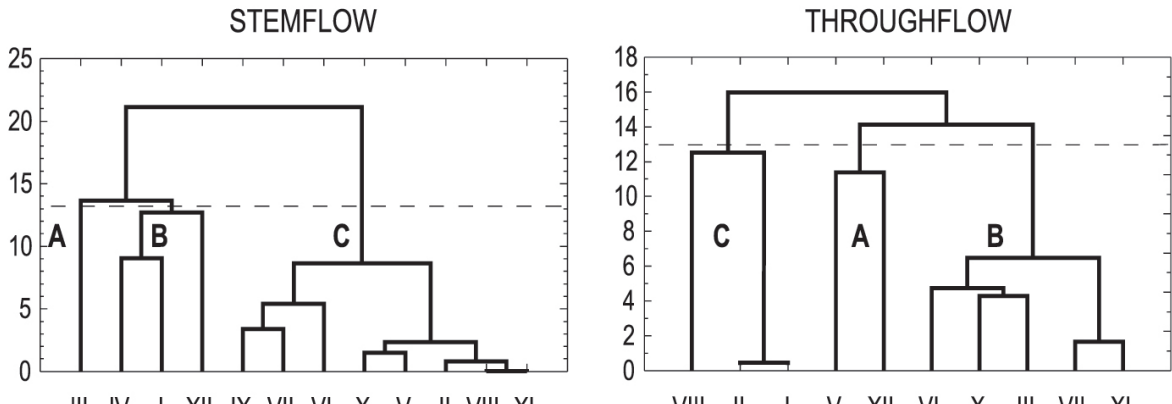

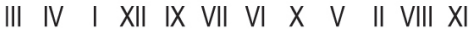

$\begin{array}{llllllllllllllll} & \text { VIII } & \text { II } & \text { I } & \text { V } & \text { XII } & \text { VI } & X & \text { III } & \text { VII } & \text { XI }\end{array}$

GROUNDWATER

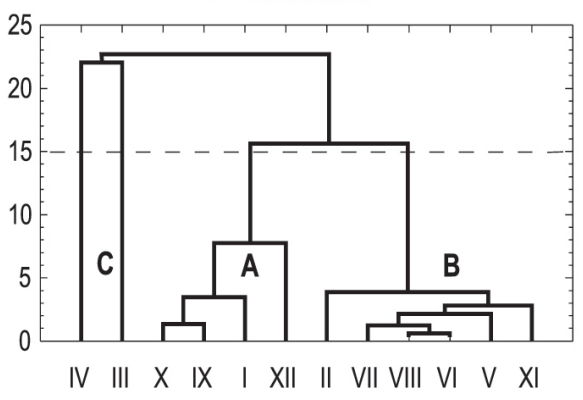

Month

LAKE WATER

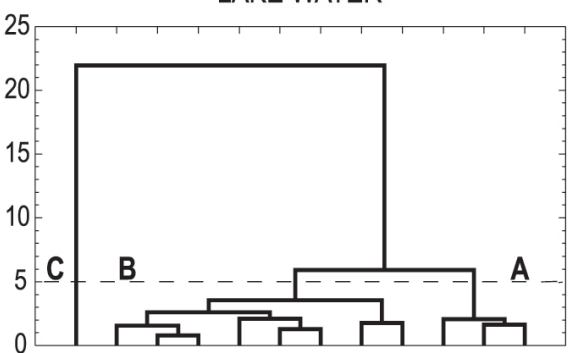

III IX VII VI VIII V IV II XII $\quad X \quad$ |

Month

Fig. 3. Seasons of mineralization of water circulating within the Lake Gardno catchment

Explanations: A - season with high water mineralization, B - season with average water mineralization, $\mathrm{C}$ - season with reduced water mineralization.

Source: authors' own study. 
Table 3. Hydrochemical seasons of concentration levels of biogenic ions $\left(\mathrm{NO}_{3}^{-}, \mathrm{NH}_{4}^{+}, \mathrm{K}^{+}\right)$ denudative ions $\left(\mathrm{SO}_{4}^{2-}, \mathrm{Ca}^{2+}, \mathrm{Mg}^{2+}\right)$ and ions derived from the supply of sea aerosols $\left(\mathrm{Cl}^{-}\right.$, $\mathrm{Na}^{+}$) within the Lake Gardno catchment

\begin{tabular}{|c|c|c|c|c|c|c|c|c|c|c|c|c|c|c|c|}
\hline \multirow{2}{*}{$\begin{array}{l}\text { Water } \\
\text { circulation } \\
\text { stage }\end{array}$} & \multicolumn{3}{|c|}{ 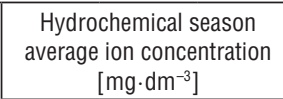 } & \multicolumn{12}{|c|}{ Time of occurrence (months) } \\
\hline & $\underset{\text { (high) }}{\mathrm{A}}$ & \begin{tabular}{|c|} 
B \\
(ave- \\
rage) \\
\end{tabular} & $\begin{array}{c}\mathrm{C} \\
\text { (low) }\end{array}$ & $\mathrm{XI}$ & XII & 1 & $\|$ & III & IV & V & VI & VII & VIII & IX & $x$ \\
\hline \multicolumn{16}{|c|}{ Biogenic ions } \\
\hline Precipitation & 7.82 & 3.54 & 1.61 & B & c & B & B & A & A & A & B & B & B & B & B \\
\hline Throughfall & 23.25 & 15.33 & 8.39 & B & B & c & B & B & B & A & B & A & B & B & B \\
\hline Stemflow & 31.21 & 20.63 & 10.71 & B & B & B & c & A & B & B & c & B & B & B & B \\
\hline Throughflow & 13.81 & 8.57 & 4.25 & C & B & C & C & B & - & A & B & c & B & - & $\mathrm{c}$ \\
\hline Groundwater & 16.54 & 9.45 & 6.50 & c & c & A & B & c & c & c & c & c & c & c & A \\
\hline Lake water & 3.24 & 2.86 & 2.25 & A & A & A & B & A & c & c & c & c & c & c & c \\
\hline \multicolumn{16}{|c|}{ Denudation ions } \\
\hline Precipitation & 3.62 & 2.76 & 2.14 & B & A & C & A & A & A & A & A & B & B & B & B \\
\hline Throughfall & 12.62 & 8.82 & 6.65 & B & A & c & B & B & A & B & c & B & c & B & B \\
\hline Stemflow & 33.60 & 25.11 & 11.87 & c & A & A & c & A & B & c & c & c & c & c & c \\
\hline Throughflow & 202.73 & 171.32 & 140.29 & B & B & B & B & B & - & A & B & B & c & - & B \\
\hline Groundwater & 335.83 & 309.67 & 290.38 & A & A & A & A & c & B & A & A & A & A & A & A \\
\hline Lake water & 271.56 & 256.56 & 238.76 & A & A & A & A & c & B & B & B & B & B & B & A \\
\hline \multicolumn{16}{|c|}{ Sea aerosols } \\
\hline Precipitation & 7.30 & 2.78 & 2.04 & C & A & C & C & B & C & B & C & C & C & C & B \\
\hline Throughfall & 30.26 & 17.38 & 9.24 & c & A & c & c & c & B & c & c & c & c & c & C \\
\hline Stemflow & 35.70 & 22.18 & 8.54 & C & B & A & B & A & A & C & C & C & C & C & c \\
\hline Throughflow & 17.87 & 10.11 & 6.16 & A & A & B & B & B & - & B & B & C & C & - & B \\
\hline Groundwater & 81.84 & 70.66 & 62.17 & B & A & B & B & c & c & B & B & B & B & B & B \\
\hline Lake water & 53.81 & 49.56 & 44.60 & A & B & A & B & C & A & A & A & A & A & A & A \\
\hline
\end{tabular}

Source: authors' own study.

average content of solutes was at $425.44 \mathrm{mg} \cdot \mathrm{dm}^{-3}$. In March and April, in the season of reduced mineralization, underground water had the total concentration of solute ions at $364.05 \mathrm{mg} \cdot \mathrm{dm}^{-3}$. Then lake water had its high contents of solutes $\left(328.80 \mathrm{mg} \cdot \mathrm{dm}^{-3}\right)$ in October, November and January. In turn, the season of reduced hydrochemical activity in Lake Gardno occurred in March (284.93 $\left.\mathrm{mg} \cdot \mathrm{dm}^{-3}\right)$. 
The seasonal dynamics of water mineralization was measured by a rate (indicator) of its irregularities which was determined by the quotient of average water mineralization of months with increased hydrochemical activity (A) to average water mineralization of months with reduced hydrochemical activity $(\mathrm{C})$. Within the Lake Gardno catchment water participating at the atmospheric stage of the water circle was characterized by the highest seasonal dynamics. Stemflow was characterized by the highest seasonal variability (2.57), which was significantly higher than in throughfall (1.73) and precipitation in the open field (1.71). At the lithospheric stage of the water cycle irregularities in water mineralization in hydrochemical seasons were much lower (for throughflow it was 1.17 , for underground and lake water 1.17 and 1.15 , respectively).

In the case of atmospheric precipitation reaching the forest zone the season of high concentration levels of biogenic ions $\left(7.82 \mathrm{mg} \cdot \mathrm{dm}^{-3}\right.$ on average $)$ occurred in spring months, from March to May. In turn, the season of reduced concentration of nutrients in atmospheric precipitation occurred in December $\left(1.61 \mathrm{mg} \cdot \mathrm{dm}^{-3}\right)$. The rest of the year saw average concentration levels of nitrate, ammonium and potassium ions $\left(3.54 \mathrm{mg} \cdot \mathrm{dm}^{-3}\right)$. For throughfall the highest concentration of biogenic elements occurred in May and July, and it was 3 times higher $\left(23.25 \mathrm{mg} \cdot \mathrm{dm}^{-3}\right)$ than in atmospheric precipitation reaching tree-tops. For stemflow concentration levels of biogenic elements in March, in the season of highest activity $\left(31.25 \mathrm{mg} \cdot \mathrm{dm}^{-3}\right)$, were 4 times higher than in the season with the highest concentration of nutrients in atmospheric precipitation. The season with reduced activity of biogenic elements at the atmospheric-forest stage of water migration occurred in January (throughfall at $8.39 \mathrm{mg} \cdot \mathrm{dm}^{-3}$ ) as well as in February and June (stemflow $10.31 \mathrm{mg} \cdot \mathrm{dm}^{-3}$ ). For throughfall and stemflow the season of average concentration levels of biogenic elements lasted 9 months. The lithospheric system of water circulation was dominated by the season of reduced activity of biogenic elements, which was particularly noticeable in underground water (for 9 months, from March to September and from November to December, with their average concentration of nutrients at $6.50 \mathrm{mg} \cdot \mathrm{dm}^{-3}$ ) and in lake water (for 7 months from April to October with their average concentration of nutrients at2.25 $\left.\mathrm{mg} \cdot \mathrm{dm}^{-3}\right)$. The season of high concentration of biogenic elements in underground water was in January and October (their average concentration at $\left.16.54 \mathrm{mg} \cdot \mathrm{dm}^{-3}\right)$ and in the Lake Gardno water - from November to January and March (their average concentration at $3.24 \mathrm{mg} \cdot \mathrm{dm}^{-3}$ ). The highest temporal variability in the activity of biogenic elements was characterised by throughflow (the highest level was in May). However, no throughflow was reported in April and September. The highest seasonal variability of biogenic elements, manifested in the difference in the concentrations of biogenic elements between the season 
with the highest and lowest hydrochemical activity, characterized stemflow $\left(20.50 \mathrm{mg} \cdot \mathrm{dm}^{-3}\right)$ and the lowest - lake water $\left(0.99 \mathrm{mg} \cdot \mathrm{dm}^{-3}\right)$ (Tab. 3$)$.

The season of high hydrochemical activity of denudative ions at the atmospheric stages of the water cycle occurred in December and spring months, especially from March to April. The concentration level of denudative ions in atmospheric precipitation was $3.62 \mathrm{mg} \cdot \mathrm{dm}^{-3}$ on average at that time, in throughfall - much higher $-12.62 \mathrm{mg} \cdot \mathrm{dm}^{-3}$ and in stemflow - very high $-33.60 \mathrm{mg} \cdot \mathrm{dm}^{-3}$. Then the period of reduced activity of denudative ions occurred in winter months, in January and February, when the concentration level of calcium, sulphate and magnesium ions ranged in total from $2.14 \mathrm{mg} \cdot \mathrm{dm}^{-3}$ in precipitation in the open field to $6.65 \mathrm{mg} \cdot \mathrm{dm}^{-3}$ in throughfall and $11.87 \mathrm{mg} \cdot \mathrm{dm}^{-3}$ in stemflow. The lithospheric system of the water circle had the longest-lasting seasons with the average and high activity of denudative ions. Underground water was characterized by the longest period of high concentration levels of denudative ions, for 10 months a year, apart from March and April, with an average concentration of $335.83 \mathrm{mg} \cdot \mathrm{dm}^{-3}$. However, lake water had the longest season of average concentration levels of denudative ions, from April to September, with an average concentration level at $256.56 \mathrm{mg} \cdot \mathrm{dm}^{-3}$.

In the case of precipitation penetrating through the forest zone the season of elevated concentration levels of ions coming from the supply of sea aerosols (chlorides and bicarbonates) was in the cool half of the year, especially in December. The aggregate average concentration of chlorides and sodium was then $>30 \mathrm{mg} \cdot \mathrm{dm}^{-3}$. High concentration levels of ions derived from the supply of sea aerosols in throughfall and stemflow were also affected by frequent advective fogs coming from over the sea at that time. In contrast, the season of low concentration levels of $\mathrm{Na}^{+}$and $\mathrm{Cl}^{-}$in throughfall and stemflow occurred in summer-autumn months, from May to October. In the case of soil and underground water the highest concentration of chloride and sodium ions was in December, however, the average concentration of these ions in soil water was 4 times lower $\left(17.87 \mathrm{mg} \cdot \mathrm{dm}^{-3}\right)$ than in the first aquifer $\left(81.84 \mathrm{mg} \cdot \mathrm{dm}^{-3}\right)$. Then the season of reduced activity of $\mathrm{Na}^{+}$ and $\mathrm{Cl}^{-}$in soil water occurred during summer months and in groundwater - during spring months. The Lake Gardno water was characterised by a very long season of elevated concentration levels of ions derived from the supply of sea aerosols, which lasted 8 months, from April to May and the average concentration of these ions was $53.81 \mathrm{mg} \cdot \mathrm{dm}^{-3}$ at that time. The season of reduced activity of chloride and sodium ions $\left(44.60 \mathrm{mg} \cdot \mathrm{dm}^{-3}\right)$ occurred in the lake in March only. 


\section{Delivery of dissolved and organic matter to soils}

The specificity of the studied coastal forested early-glacial geoecosystems is determined by a large supply of solutes together with fog-generated sediments and by large loads of biogenic elements reaching the land area.

A large quantity of highly mineralized water with fog is a characteristic feature of the Lake Gardno catchment and its coastal location. Advection fogs coming from the sea provide considerable loads of solutes, especially sea aerosols in the form of chloride and sodium ions with their share in the mineralization of fog-generated sediments at $45 \%$. On average, every year, $11.54 \mathrm{~g} \cdot \mathrm{m}^{-2} \mathrm{Cl}^{-}$- ions and $4.98 \mathrm{~g} \cdot \mathrm{m}^{-2}$ $\mathrm{Na}^{+}$ions were supplied with fog. The remainder was made by biogenic ions at $37 \%\left(13.58 \mathrm{~g} \cdot \mathrm{m}^{-2}\right)$ and denudative ions at $18 \%\left(6.61 \mathrm{~g} \cdot \mathrm{m}^{-2}\right)$. Out of biogenic ions the highest supply referred to $\mathrm{NO}_{3}^{-}$ions $\left(11.94 \mathrm{~g} \cdot \mathrm{m}^{-2} \cdot \mathrm{a}^{-1}\right)$ and out of denudative ions - to $\mathrm{SO}_{4}^{2-}$ ions $\left(3.47 \mathrm{~g} \cdot \mathrm{m}^{-2} \cdot \mathrm{a}^{-1}\right.$, Fig. 4). On average, every year, $84.5 \mathrm{~mm}$ of water was supplied to the Lake Gardno geoecosystem with fog.

The Lake Gardno catchment is characterised by its almost complete forestation and consequently significant loads of biogenic elements reaching the ground. The supply of biogenic matter was mostly affected by fog-generated sediments which provided $72 \%$ nitrates, $68 \%$ ammonium ions and $33 \%$ potassium.

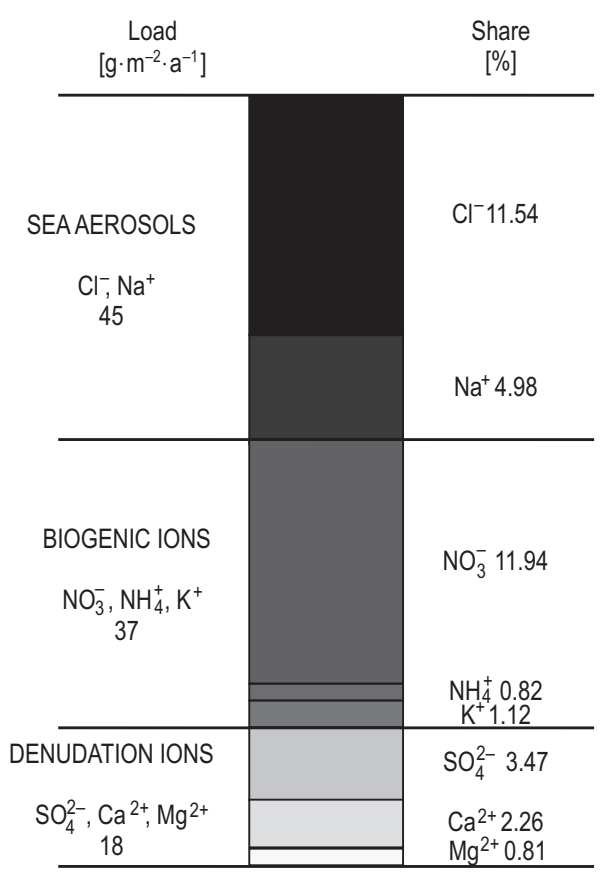

Throughfall provided $25 \%$ nitrates, $27 \%$ ammonium ions and $57 \%$ potassium. There was a relatively low supply of biogenic elements in stemflow: only $3 \%$ nitrates, $5 \%$ ammonium ions and $10 \%$ potassium. On average, every year, $13.87 \mathrm{~g} \cdot \mathrm{m}^{-2}$ biogenic elements was provided with fog. The deposition of biogenic elements with throughfall was twice lower $\left(6.46 \mathrm{~g} \cdot \mathrm{m}^{-2}\right)$ and with stemflow - 15 times lower $\left(0.91 \mathrm{~g} \cdot \mathrm{m}^{-2}\right.$, Fig. 5$)$.

Fig. 4. Load $\left[\mathrm{g} \cdot \mathrm{m}^{-2} \cdot \mathrm{a}^{-1}\right]$ and share $[\%]$ levels of biogenic, denudative ions and sea aerosols in the supply of solutes with fog within the Lake Gardno catchment (2010-2014) in a beech stand Source: authors' own study. 


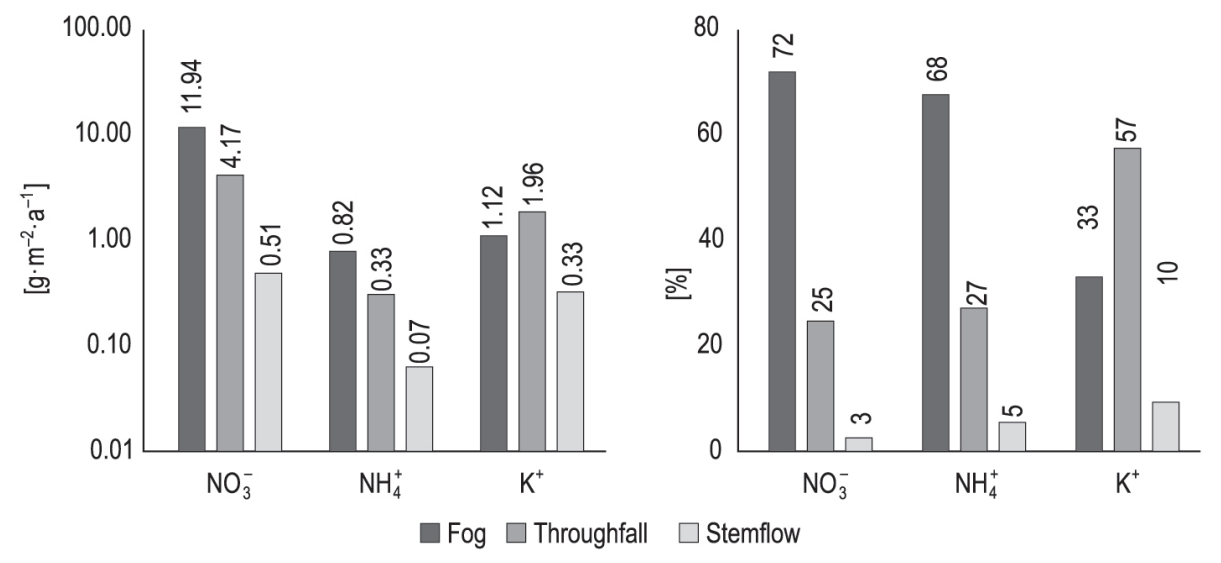

Fig. 5. Load $\left[\mathrm{g} \cdot \mathrm{m}^{-2} \cdot \mathrm{a}^{-1}\right]$ and share [\%] levels of biogenic $\left(\mathrm{NO}_{3}^{-}, \mathrm{NH}_{4}^{+}, \mathrm{K}^{+}\right)$in the supply of solutes with throughfall, stemflow and fog into the Lake Gardno catchment (2010-2014) in a beech stand

Source: authors' own study.

In 2010-2014 there were some measurements on the deposition of organic matter and its rates conducted within the catchment. On a monthly basis samples were collected from 15 collectors of organic precipitation with a total intake area of $0.8 \mathrm{~m}^{2}$. The annual production of dry organic fallout from trees for the Lake Gardno catchment in 2010-2014 amounted to $2.9 \mathrm{t} \cdot \mathrm{ha}^{-1}$. The lowest supply (1.9 $\left.\mathrm{t} \cdot \mathrm{ha}^{-1}\right)$ was recorded in 2012 and the highest $\left(4.5 \mathrm{t} \cdot \mathrm{ha}^{-1}\right)$ in 2011 . The supply was mainly made by beech leaves which accounted for the annual average supply of $1.6 \mathrm{t} \cdot \mathrm{ha}^{-1}$ of dry organic matter. Branches, bud scales, bark provide $1.1 \mathrm{t} \cdot \mathrm{ha}^{-1}$ of dry organic matter. The lowest supply was made by fruit $\left(0.18 \mathrm{t} \cdot \mathrm{ha}^{-1}\right)$ and needles $\left(5.1 \mathrm{~kg} \cdot \mathrm{ha}^{-1}\right)$.

\section{Specificity of the chemical composition of circulating water}

The hydrodynamic system of the examined catchment covers all processes involved in the circulation of water as the main factor of landscape changes. It is characterized by a series of linear and non-linear dependencies with the environment. The hydrodynamic system of the catchment is subject to transformations under the influence of external supplies of energy and matter and as a result of changes over time within the system. Supplies of energy and matter include solar radiation, atmospheric precipitation and deposits with their accompanying pollution of 


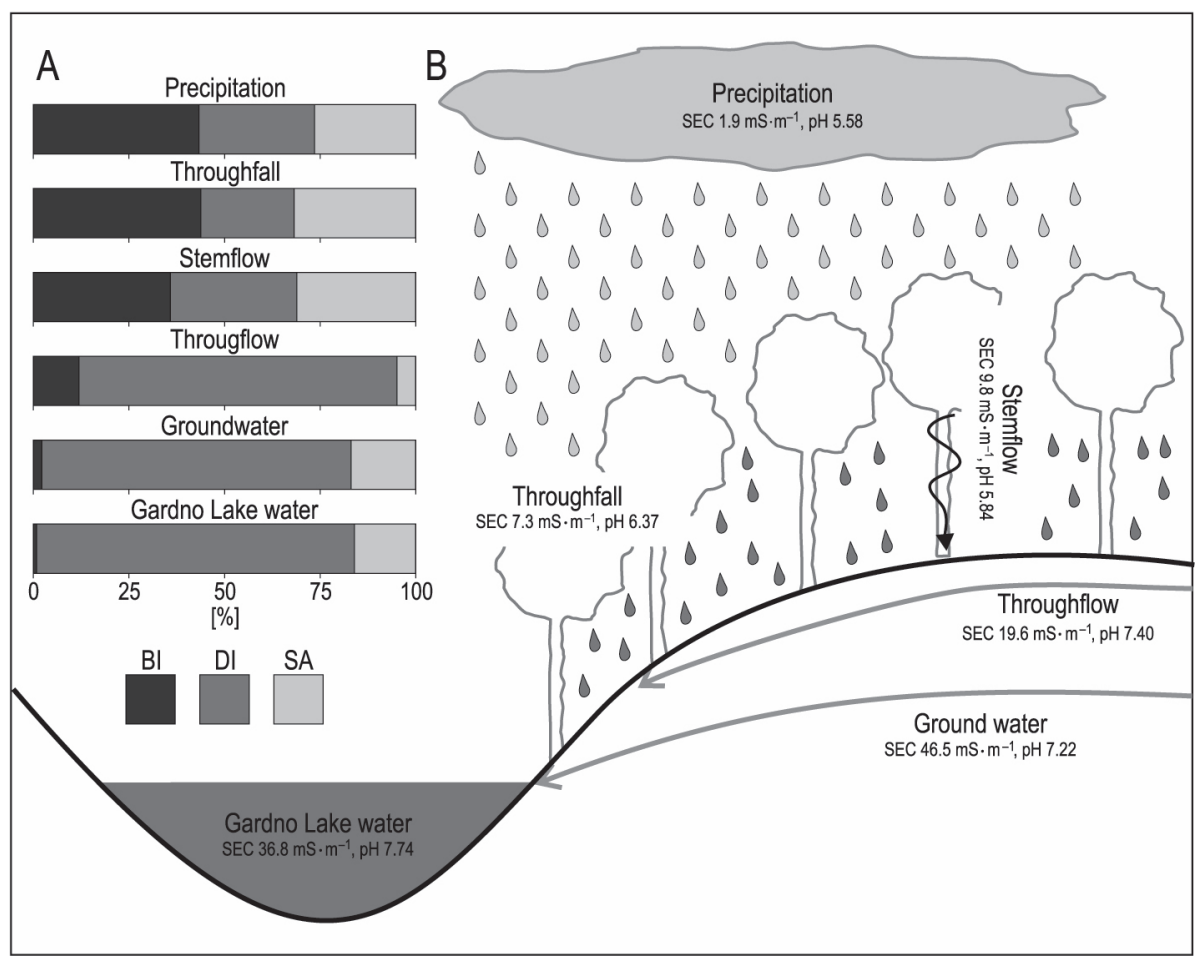

Fig. 6. Model of the transformation of atmospheric precipitation within the Lake Gardno catchment

Explanations: A - chemistry of the water at various stages of the cycle, B - stages of the water cycle, BI - biogenic ions, DI - denudation ions, SA - sea aerosols, SEC - specific electrical conductivity, $\mathrm{pH}-$ reaction.

Source: authors' own study.

water as well as mineral and organic matter delivered by other processes. External stimuli and local conditions lead to transport and transformation of atmospheric precipitation within the catchment. Depending on the kind of impact of individual environmental components, water is depleted in or enriched with adequate ions. It leads to changes in its impact onto the geoecosystem at subsequent stages of the circle.

It was possible to diagnose Lake Gardno and its operation on the grounds of long-term research studies on water quality and quantity at every stage of this circle (Fig. 6B). The following stages of water circulation were singled out: atmospheric (atmospheric precipitation, throughfall, stemflow), transitional (throughflow) and 
lithospheric (underground water, lake water). Within the Lake Gardno catchment, on a monthly basis, water with its quantitative aspects and physicochemical properties was examined at every stage of its circulation.

This systematic monitoring of the water cycle allowed to determine the physicochemical transformation of atmospheric precipitation in the catchment and percentage levels of biogenic, denudative ions and sea aerosols in the total water mineralization at individual stages of circulation within the catchment (Fig. 6A).

\section{Conclusion}

The coastal location within quaternary forms, considerable altitude variances, good permeability of surface formations, non-run-off surface and almost total forestation (mainly with the beech stand) determine the specifics of the Gardno Lake catchment.

The geographic individuality of the Gardno Lake geoecosystem determines the dynamics of its circulation of water and solutes:

- High temporal and spatial variability of hydrochemical seasons. Seasons of water mineralization and concentration of biogenic, denudative ions and ions from sea aerosols are characterised by minor similarities at individual stages of the water cycle. Particularly outstanding variances in the annual distribution of hydro-chemical seasons relate to the atmospheric (atmospheric precipitation, throughfall and stemflow) and lithospheric (underground and lake water) stages of the water circulation. Also, the seasonal distribution of concentration levels of nutrients, denudative ions and ions derived from the supply of marine aerosols is characterised by significant variation in the year;

- Due to the almost complete forestation, within the considered geoecosystem, slope covers are reached by substantial loads of organic matter $\left(280 \mathrm{~g} \cdot \mathrm{m}^{-2} \cdot \mathrm{a}^{-1}\right.$ on average). Moreover, the geochemical transformations of these surface formations are mostly affected by loads of biogenic elements provided with throughfall and stemflow $\left(7.5 \mathrm{~g} \cdot \mathrm{m}^{-2} \cdot \mathrm{a}^{-1}\right.$ on average $)$;

- Due to the influx of fogs from over the sea within the analysed geoecosystem the water and denudation balance is significantly impacted by a supply of water from fogs $\left(85 \mathrm{~mm} \cdot \mathrm{a}^{-1}\right.$ on average $)$ and loads of solutes $\left(37 \mathrm{~g} \cdot \mathrm{m}^{-2} \cdot \mathrm{a}^{-1}\right.$ on average $)$. The above-mentioned general principles of operation of the Gardno Lake catchment at the Wolin Island can be considered representative to similar geoecosystems of the coastal early-glacial zones in Poland (Cieśliński 2003, 2004; Cieśliński, Major 2012). 


\section{Acknowledgements}

The study was co-financed by the NCN research project no. N N304 274340 entitled: "The current state and functioning of the natural environment within the selected areas of West Pomerania region under climatic changes and increased anthropopressure”.

\section{References}

Adamczewska M., Jewasińska L., 1986, Gleby Wolinskiego Parku Narodowego, [in:] A. Kostrzewski (ed.), Wolinski Park Narodowy. Monografia geograficzna, Poznań, 136-142. Bogucki J., Tamulewicz J., 1992, Topoklimat, [in:] A. Kostrzewski (ed.), Charakterystyka fizyczno-geograficzna geoekosystemów wodnych Wolinskiego Parku Narodowego, maszynopis, Poznań, 33-37.

Borowiec S., 1961, Gleby brunatne i bielicowe na terenie Wolinskiego Parku Narodowego oraz powiqzania ich z siedliskowymi typami lasu, SYLWAN 105, 7, 19-30.

Borowiec S., 1969, Zagadnienia gleboznawczo-siedliskowe Wolińskiego Parku Narodowego, Szczecińskie Towarzystwo Naukowe, Wydział Nauk Przyrodniczo-Rolnych, 31, 3, Szczecin.

Borowiec S., 1994, Wyniki i perspektywy badań gleboznawczych na terenie Wolinskiego Parku Narodowego, Klify, 1, 25-32.

Choiński A., 1978, Hydrografia jezior wolińskich, [in:] A. Kostrzewski (ed.), Studia z geografii fizycznej i ekonomicznej wyspy Wolin, SKNG UAM, Poznań, 93-106.

Cieśliński R., 2003, The abiotic conditions of deltaic lake ecosystem (on the example of the Druzno Lake catchment), Journal of Water and Land Development, 7, 33-47.

Cieśliński R., 2004, Chemical classifications of waters of chosen lakes of the central coast of the Polish coastline zone of the Southern Baltic, Limnological Review, 4, 57-66.

Cieśliński R., Major M., 2012, Differences in the abiotic parameters of water in coastal lakes in the light of the EU Water Framework Directive: An example of the polish southern Baltic coast, Acta Geophysica, 60, 4, 1159-1179.

Gościńska M., 2003, Charakterystyka litologiczna zlewni jeziora Gardno na wyspie Wolin, [in:] A. Kostrzewski (ed.), Wolinski Park Narodowy, środowisko przyrodnicze ksætattowanie i ochrona, SKNG, Poznań, 19-30.

Kolander R., 2005, Źródta dostawy i drogi przeptywu materii nierozpuszczonej w bukowych siedliskach leśnych wyspy Wolin, [in:] R.K. Borówka (ed.), Plejstoceńskie i holoceńskie przemiany śodowiska przyrodniczego Polski, Szczecin, 38-43.

Kolander R., Kostrzewski A., Tylkowski J., 2008, Seasonal variations in the chemical composition of water circulating in the Lake Gardno catchment on Wolin Island, Quaestiones Geographicae, 27A/2, Series A, Wydawnictwo Naukowe UAM, 21-27.

Kolander R., Tylkowski J., 2007, Sezonowa smienność mineralizacji wód krqäqcych w zlewni jeziora Gardno na wyspie Wolin jako podstawa wydzielenia sezonów hydrochemicznych, [in:] A. Kostrzewski, A. Andrzejewska (eds.), Zintegrowany Monitoring Środowiska 
Przyrodniczego, Program Zintegrowanego Monitoringu Środowiska Przyrodničego a zadania obszarów Natura 2000, Biblioteka Monitoringu Środowiska, Warszawa, 331-338.

Kolander R., Tylkowski J., 2008, Hydrochemical seasons in the Lake Gardno catchment on Wolin Island (north-western Poland), Limnological Reviev, 8, 1-2, 27-34.

Komisarek J., Marcinek J., Kozłowski M., Wiatrowska K., Pawłowski M., Kunz T., Wytykowska-Sroka E., 2010, Kartowanie glebowe w Stacji Bazowej ZMŚP „Biała Góra”, maszynopis, Poznań.

Kostrzewski A. (ed.), 1978, Studia z geografii fizycznej i ekonomicznej wyspy Wolin, SKNG UAM, Poznań.

Kostrzewski A. (ed.), 1993, Geoekosystem obszarów nizinnych, PAN, Zeszyty Naukowe, 6, Wrocław-Warszawa-Kraków.

Kostrzewski A., Kruszyk R., Kolander R., 2006, Zintegrowany Monitoring Środowiska Przyrodniczego. Zasady organizacji, system pomiarowy, wybrane metody badań, http://www. staff.amu.edu.pl/ zmsp/wyt2006/wyt2006.html (18.08.2016).

Kostrzewski A., Samołyk M., Tylkowski J., 2015, Physical geographic conditions in the Gardno Lake catchment (Wolin Island), Prace Geograficzne, 143, 65-84.

Kostrzewski A., Tylkowski J., Samołyk M., 2011, Indywidualnośćprzyrodnicza funkcjonowania zlewni Jeziora Gardno (wyspa Wolin), [in:] A. Kostrzewski, M. Samołyk (eds.), Zintegrowany Monitoring Środowiska Przyrodniczego. Funkcjonowanie geoekosystemów w warunkach smian ü̇ytkowania terenu i narastajqcej antropopresji, Biblioteka Monitoringu Środowiska, XXVIII, Biała Góra, 121-137.

Kruszyk R., Kostrzewski A., Tylkowski J., 2015, Variability of throughfall and stemflow deposition in pine and beech stands (Czarne Lake catchment, Gardno Lake catchment on Wolin Island), Prace Geograficzne, 143, 55-102.

Piotrowska H., 1955, Zespoły leśne wyspy Wolin, Prace Komisji Biologicznej PTPN, 16, 5, Poznań, 1-168.

Prusinkiwicz Z., 1971, Naspy przyklifowe - nowy typ gleb morskiego pobrze:a, Zeszyty Naukowe UMK, Geografia, 26, 133-157.

Tylkowski J., 2012, Zmienność cæasowa i przestræenna warunków termicæno-opadowych strefy br:egowej Zatoki Pomorskiej, [in:] A. Kostrzewski, J. Szpikowski (eds.), Zintegrowany Monitoring Środowiska Przyrodniczego. Funkcjonowanie geoekosystemów w różnych strefach krajobrawowych, Biblioteka Monitoringu Środowiska, XXIX, Storkowo, 209-219.

Tylkowski J., 2013, Temporal and spatial variability of air temperature and precipitation at the Polish coastal zone of southern Baltic Sea, Baltica, 26, 1, 83-94.

Tylkowski J., Kolander R., Kostrzewski A., 2008, Transformacja opadów atmosferycznych w zlewni Jeziora Gardno na wyspie Wolin, [in:] K. Rotnicki, J. Jasiewicz, M. Woszczyk (eds.), Holoceńskie præemiany wybræę̇y $i$ wód potudniowego Battyku, Wydawnictwo Tekst, Poznań-Bydgoszcz, 139-143.

Tylkowski J., Kolander R., Kostrzewski A., 2009, Sezonowa smienność dostawy biogenów do zlewni Jeziora Gardno, wyspa Wolin, [in:] W. Bochenek, M. Kijowska (eds.), Zintegrowany 
Monitoring Środowiska Przyrodniczego, Funkcjonowanie Środowiska przyrodniczego w okresie præemian gospodarczych w Polsce, Biblioteka Monitoringu Środowiska, Szymbark, 258-267. Tylkowski J., Samołyk M., 2010, Monitoring fizykochemicænych właściwości pokrywy śnieżnej wyspy Wolin, Monitoring Środowiska Przyrodniczego, Kieleckie Towarzystwo Naukowe, Kielce, 11, 73-80.

Tylkowski J., Samołyk M., 2015, Meteorological conditions, physiochemical properties, thermal-oxygen stratification, water overturn and water balance of Lake Gardno on Wolin Island, Limnological Review, 15, 3, 107-118.

Andræej Kostræeweski

Adam Mickiewicz University in Poznań

Institute of Geoecology and Geoinformation

10 Bogumita Krygowskiego Str., 61-680 Poznań, Poland

e-mail:anko@amu.edu.pl

Jacek Tylkowski

Adam Mickiewicz University in Poznan

Institute of Geoecology and Geoinformation

10 Bogumita Krygowskiego Str., 61-680 Poznañ, Poland

e-mail:jatyl@amu.edu.pl

Marius: Samotyk

Adam Mickiewicz University in Poznań

Institute of Geoecology and Geoinformation

10 Bogumita Krygowskiego Str., 61-680 Poznań, Poland

e-mail:mars@amu.edu.pl 